\title{
Systemic Approach to Prevent Inadvertent Perfusion in Eyes with Extensive Choroidal Detachment, Suprachoroidal Fluid, and Hypotony During Pars Plana Vitrectomy
}

\author{
Zhaotian Zhang - Dong Fang - Manjuan Peng - Yantao Wei • \\ Li Wang · Shuxin Fan · Shaochong Zhang (D)
}

Received: August 28, 2018 / Published online: November 24, 2018

(C) The Author(s) 2018

\section{ABSTRACT}

Introduction: Rhegmatogenous retinal detachment (RRD) with extensive choroidal detachment (CD), suprachoroidal fluid (SCF), and lower intraocular pressure (IOP) markedly increase the difficulty of pars plana vitrectomy (PPV) because it makes the insertion and placement of the trocar cannulas for PPV timeconsuming and technically challenging. In this study, we developed a systemic approach that avoids the risks of sclerotomy or using a longer trocar to help in the safe placement of the trocar cannulas in order to prevent inadvertent fluid/ air perfusion during PPV in RRD eyes with extensive CD, SCF, and hypotony.

Methods: This is a prospective case series study. Consecutive patients diagnosed with RRD with extensive $\mathrm{CD}, \mathrm{SCF}$, and hypotony were recruited. The main steps included injecting transconjunctival intravitreal fluid to increase IOP and enable the passive egress of SCF into

Enhanced digital features To view enhanced digital features for this article go to https://doi.org/10.6084/ m9.figshare.7127138.

Zhaotian Zhang and Dong Fang contributed equally.

Z. Zhang · D. Fang - M. Peng · Y. Wei · L. Wang ·

S. Fan · S. Zhang ( $\square)$

State Key Laboratory of Ophthalmology, Zhongshan

Ophthalmic Center of Sun Yat-sen University,

Guangzhou, China

e-mail: zhangshaochong@gzzoc.com the subconjunctival space, creating and broadening the inner opening for the trocar cannulas, transillumination with an endoillumination light pipe, and indentation of the infusion cannula to create maximum visual exposure of the inner segment and help peripheral shaving around the infusion tip. Main outcomes: the success rate of the infusion placement into the vitreous cavity, surgical duration, intra- and postoperative complications, and functional and anatomical outcomes.

Results: Thirty-eight patients (38 eyes) were included. The first-time success rate of cannula placement into the vitreous cavity was $100 \%$, with a mean surgical duration of $8.5 \pm 1.8 \mathrm{~min}$. No intra- or postoperative complications related to the approach were observed. Significant vision improvement was noted in $68.4 \%$ (26/ 38 ) of the patients at the final follow-up. The primary and final retinal reattachment rates were $94.7 \%(36 / 38)$ and $100 \% \quad(38 / 38)$, respectively.

Conclusion: This systemic approach is a feasible option to ensure the safe placement of trocar cannulas and prevent inadvertent perfusion during small-gauge PPV in RRD eyes with extensive $\mathrm{CD}$, SCF, and hypotony, without the need for a draining sclerotomy, a longer trocar, or a sharp clearing of the cannula tip.

Keywords: Choroidal detachment; Ciliary detachment; Lower intraocular pressure/ 
hypotony; Ophthalmology; Pars plana vitrectomy; Rhegmatogenous retinal detachment; Suprachoroidal fluid; Trocar

\section{INTRODUCTION}

Extensive choroidal detachment (CD) associated with rhegmatogenous retinal detachment (RRD) is an unfavorable condition for surgical intervention [1-3]. The presence of suprachoroidal fluid (SCF) and lower intraocular pressure (IOP) are two concomitant abnormalities with CD-RRD [2, 4-6]. Anatomically, it makes the insertion and placement of trocar cannulas for pars plana vitrectomy (PPV) timeconsuming and technically challenging. Various adjuvant techniques have been proposed to solve these problems, including the administration of steroids, sclerotomy to create passive drainage of the SCF, increasing IOP with an intravitreal injection of balanced saline solution (BSS), and penetration with a longer trocar (6 mm) [7-13]. The theoretical risks include the side effects of the drug and iatrogenic trauma of the transparent lens, which might consequently increase surgical difficulty and worsen patient prognosis. Draining the sclerotomy creates unnecessary trauma to the sclera and the underlying choroidal tissue. Secondary choroidal hemorrhage can occur after that, especially when the eyes are hypotonic. Additionally, longer trocars are not always readily available in certain areas of the world (including the authors' institution as well as most ophthalmic institutions in China).

In the era of microincision vitrectomy (MIV), how can we maximize the usability of the trocar cannula to solve the aforementioned problems? With the introduction of MIV systems, the trocar cannula has enabled us to perform infusion placement more safely and effectively [14-16]. However, the concomitant presence of extensive CD, SCF, and hypotony appears to be a major barrier to surgeons starting PPV because the surgeon has to make extra efforts to ensure the trocar cannula sufficiently penetrates the vitreous cavity; otherwise, inadvertent perfusion of fluid/air can occur. Inadvertent perfusion is a serious intraoperative complication during PPV. Inadvertent perfusion refers to a series of adverse events during PPV when the infusion of fluid/air is under the open status, such as fluid/air injection into the subretinal/suprachoroidal space and slippage of the infusion cannula from the sclera tunnel. As far as we know, the details of the proposed techniques vary a great deal, which makes the process difficult for other surgeons to master and achieve clear outcomes. To the best of our knowledge, there has not been any stepwise and systemic approach to solve the problems based only on the trocar cannula system. Therefore, we have developed a systemic approach that does not use a draining sclerotomy or longer trocar to help with the safe placement of trocar cannulas, thus preventing inadvertent fluid/air perfusion during PPV in RRD eyes with extensive $\mathrm{CD}, \mathrm{SCF}$, and hypotony.

\section{METHODS}

\section{Subjects}

This study was approved by the institutional research committee of the Zhongshan Ophthalmic Center of Sun Yat-sen University (Guangzhou, China) and performed in accordance with the World Medical Association's Declaration of Helsinki. Written informed consent was obtained from each patient. The current study was an academic conclusion to share our surgical team's clinical performance and was designed as a consecutive case series study. Thus, trial registration was not required.

The patients who met the following inclusion criteria were prospectively recruited: diagnosed with RRD (confirmed by ultra-B scanning and dilated fundoscopy) (Fig. 1) with preoperative hypotony (IOP $\leq 9.0 \mathrm{mmHg}$; measured by non-contact tonometry), the existence of extensive SCF, and ciliary/choroidal detachment (confirmed by ultra-B scanning and ultrasound biomicroscopy) (Fig. 2a, b). Exclusion criteria: significant opacity of the visual axis caused by disease of the anterior segment (except for cataracts); postoperative follow-up of less than 3 months, and other serious eye diseases. 


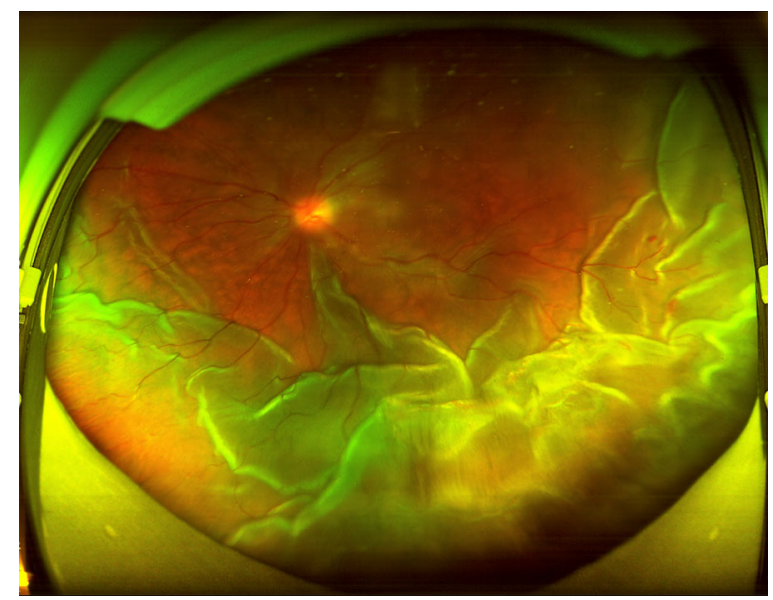

Fig. 1 Wide-angle fundoscopy revealed RRD with extensive elevation of the underlying choroidal

\section{Surgical Techniques}

All the surgeries were performed under local anesthesia by two experienced surgeons (Z.Z. and S.Z.) at Zhongshan Ophthalmic Center (ZOC) of Sun Yat-sen University, Guangzhou, China. Standard 3-port, 25-gauge PPV (Constellation Vitrectomy System, Alcon Laboratories, Fort Worth, TX) and wide-angle viewing systems (Carl Zeiss Meditec AG, Jena, Germany) were used for all of the cases. During the vitrectomy, the cutting rate was set to 5000 cuts/ min (duty cycle $=50 / 50$ ), with an infusion pressure ranging from 25 to $35 \mathrm{mmHg}$. In order to make this systemic approach brief and easy to follow, it is described step by step, as follows:
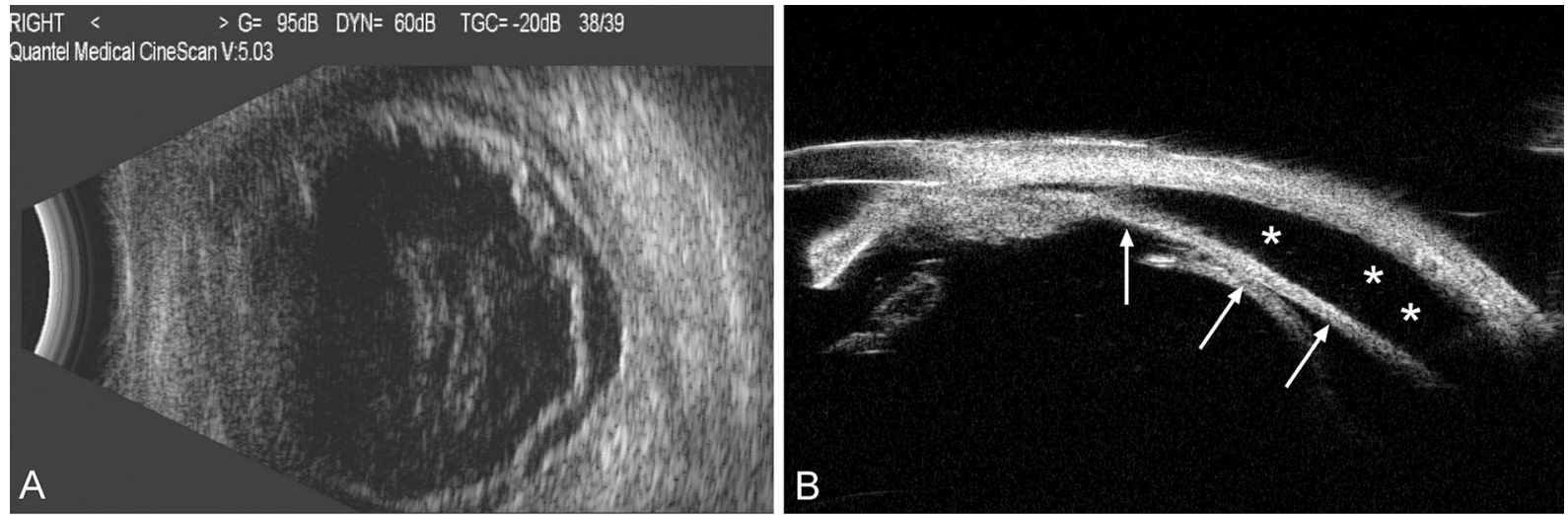

Fig. 2 a Ultra-B scanning revealed extensive retinal detachment and CD; b ultrasound biomicroscopy revealed the existence of SCF (white asterisks) and ciliary/choroidal detachment (white arrows) 

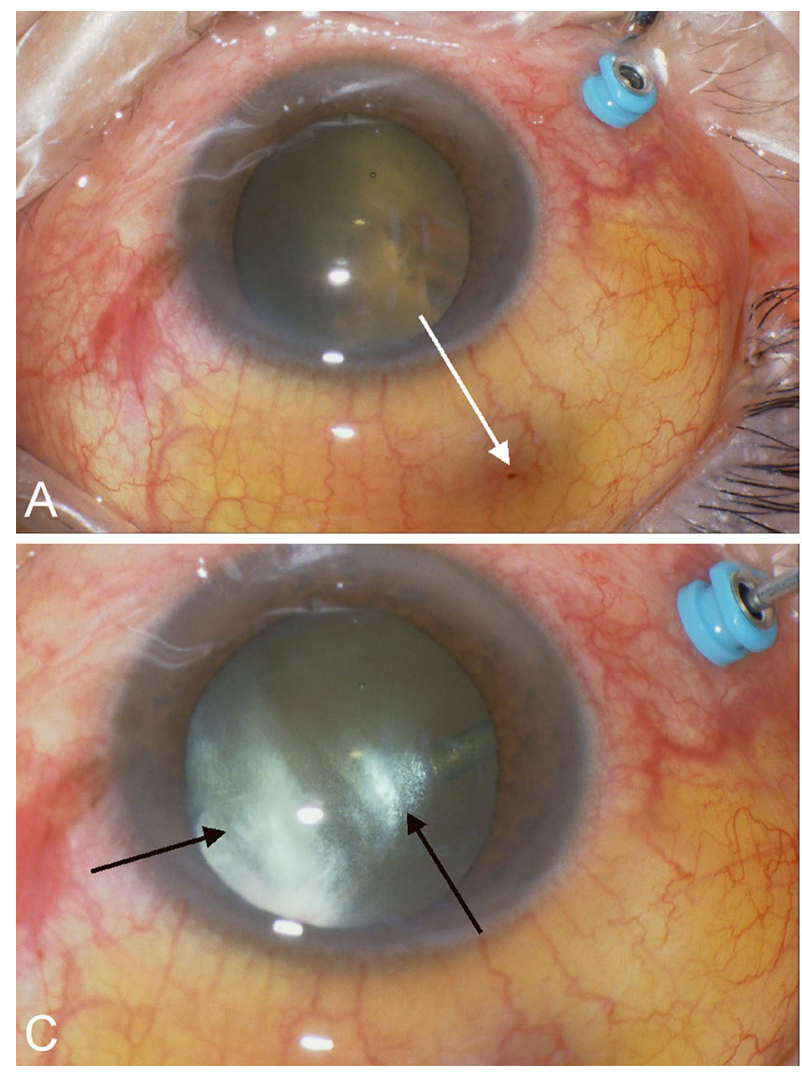

Fig. 3 Representative snapshots of surgical videos: a passive egress of SCF into the subconjunctival space from the injection site (white arrow); $\mathbf{b}$ the infusion cannula has not penetrated well into the vitreous cavity and is partially surrounded by choroidal tissues (white arrow);

Equipment CO, Ltd., Shanghai, China) was introduced to make the BSS injection through the infusion cannula. The main objective was to broaden the inner opening, thus preventing the risk of sharp iatrogenic trauma of the vitreous base.

5. Transilluminate the Infusion Cannula. The endoillumination light pipe was an ideal instrument to inspect whether the inner opening of the infusion cannula had been successfully created. Additionally, the surgeon was able to judge whether the inner opening was surrounded by condensed intraocular tissues according to the light emitting through the pupil area (Fig. 3c).

6. Connect the Infusion Line Without Switching on the Fluid Infusion. After the inner opening of the infusion cannula had been created,
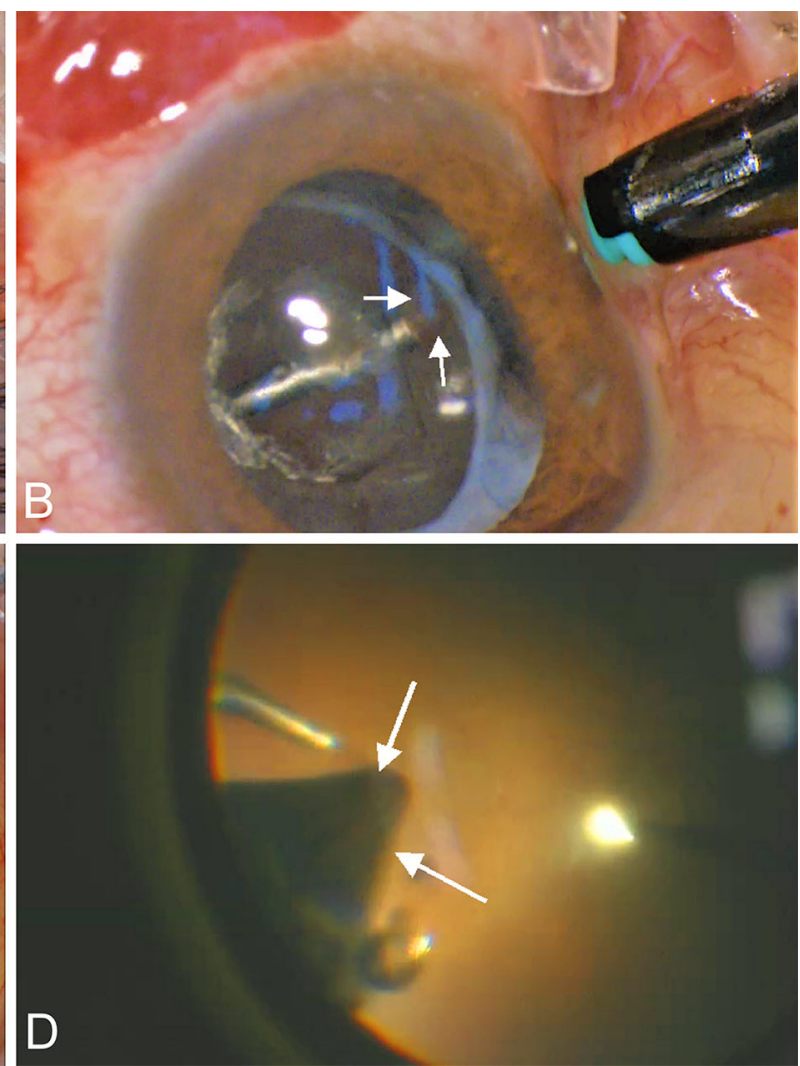

c transillumination reveals condensed intraocular tissues around the infusion cannula (black arrow); $\mathbf{d}$ the inner opening of the upper trocar cannula is completely covered inside by choroidal tissues (white arrow); crude introduction of the intravitreal instruments might be accidental

we connected the infusion line to the cannula. It is strongly recommended not to start the fluid infusion in the current step because complete penetration of the inner opening of the infusion cannula cannot be confirmed. The possible existence of condensed intraocular tissues around the inner opening prevents fluid infusion during vitrectomy.

7. Insert the Upper Trocars and Repeat Steps 3 and 4 . The objective of this step was to ensure that the endoillumination light pipe and vitrectomy probe had an enough passage for intravitreal manipulations. If the cannula tip was covered by the choroidal tissues (Fig. 3d), repeated in-and-out movement of the intravitreal instruments will push or drag the pars plana epithelium or 
vitreous base ahead of them, causing iatrogenic ora serrata dialysis and/or retinal breaks.

8. Indentation and Peripheral Shaving of the Infusion Cannula. After introduction of the vitrectomy probe and light pipe, the assistant created an indentation of the infusion cannula centripetally with forceps (without switching on the fluid infusion). When shaving the peripheral vitreous around the infusion cannula, proliferative strands/ membranes (if present) should be shaved off together. Any detached choroidal tissues (if present) covering the tip of the cannula can simultaneously be pushed backwards to the eye wall with the vitrectomy probe to completely liberate the infusion cannula in the vitreous cavity. The indentation enables maximal exposure of the inner segment of the infusion cannula in the vitreous cavity. Additionally, it maintains IOP when there is peripheral vitreous shaving around the cannula as infusion has not been started. Finally, the fluid infusion was turned on and the indentation of the infusion cannula was withdrawn. Thereafter, the surgeon continued with the routine procedures to achieve retinal reattachment. Surgical details are demonstrated in the video (Supplemental Digital Content https://doi.org/ 10.6084/m9.figshare.7127138).

\section{Outcome Measures}

Follow-up examinations were scheduled at 1 day, 1 week, 1 month, 3 months, and no less than 6 months after surgery. All eligible patients underwent comprehensive ophthalmologic examinations during the entire follow-up, including Snellen best-corrected visual acuity (BCVA) tests, non-contact tonometry, slit-lamp microscopy, and dilated fundoscopy. The main outcomes included the first-time success rate of complete penetration of the infusion trocar into the vitreous cavity, the time duration to perform the systemic approach, intra- and postoperative complications, postoperative BCVA, and primary and secondary rates of retinal reattachment.

\section{Statistical Analysis}

All data were analyzed using SPSS 19.0 statistical software (SPSS Inc., Chicago, IL, USA). Continuous and categorical variables were described as mean \pm standard deviation and proportion (\%), respectively. For continuous measurement data, the two-sample $t$ test or Mann-Whitney test was used to compare the differences, as appropriate. All Snellen visual acuity values were converted to the logarithm of the minimum angle of resolution (logMAR) for statistical analysis. The visual acuity of light perception was assigned as 2.9, hand movements (HM) as 2.6, and counting fingers as 2.3. Postoperative vision improvement was defined as a postoperative gain of two or more Snellen lines of visual acuity or a postoperative change of ambulatory vision to no less than Snellen 20/400. A twosided $P$ value less than 0.05 was considered statistically significant.

\section{RESULTS}

Thirty-eight eye patients (38 eyes, phakia/ pseudophakia $=20 / 18 ; \quad$ male $/$ female $=23 / 15$ ) were included, with a mean age of $46.4 \pm 13.9$ years (20-74 years). The mean preoperative IOP was $6.4 \pm 1.4 \mathrm{mmHg}$ (5-9 mmHg). The mean preoperative logMAR BCVA was $1.623 \pm 1.587$ (Snellen equivalent range, HM-20/100). The mean logMAR BCVA at the final follow-up was $0.830 \pm 0.486$ (Snellen equivalent range 20/2000-20/40), with a statistically significant difference when compared to the preoperative measurements $(P<0.001)$; $68.4 \%(26 / 38)$ of the patients gained significant vision improvement at the final follow-up. The first-time success rate of complete penetration of the infusion cannula was $100 \%$, without any case experiencing inadvertent perfusion of BSS/ air during all the surgical procedures. The mean surgical duration to perform the whole systemic approach was $8.5 \pm 1.8 \mathrm{~min}$ (range 6.5-12.0 $\mathrm{min}$ ). Other intraoperative complications were not observed, such as eye collapse, choroidal hemorrhage, trauma to the lens, and iatrogenic retinal breaks. No related postoperative complications were found. The primary and 
final retinal reattachment rates were 94.7\% (36/ 38 ) and $100 \%$ (38/38), respectively.

\section{DISCUSSION}

In the current article, we have introduced a systemic approach to achieving complete insertion of the infusion cannula for both phakic and pseudophakic eyes with extensive CD, SCF, and hypotony. The pathogenic mechanism of the associated CD in RRD (CD-RRD) patients has been the focus of many studies, and hypotony and inflammation have been revealed as two significant factors $[2,4-6,17,18]$. The incidence of CD-RRD was reported to be 2.0-18.79\%; the risk factors include old age, high myopia/longer axial length, aphakia/ pseudophakia, hypotony, and macular holes $[1,2,4,19]$. Regardless of the mechanism and risk factors, the current approach aimed to solve the three concomitant surgical challenges (CD, SCF, and hypotony), which surgeons sometimes must resolve before vitrectomy can be safely performed.

It is understandable from an anatomical perspective why the existence of these three concomitant conditions may markedly increase surgical difficulty and increase the risk of sclerotomy $[6,9,10,13]$. In the case of hypotony, the trocar cannula cannot easily penetrate the vitreous cavity because of the lack of sufficient back force from the eye wall. Choroidal hemorrhage and iatrogenic trauma to the lens can happen after the collapse of the eye wall during trocar insertion. The SCF accumulating at the pars plana limits maximal exposure of the infusion tip in the vitreous cavity, or it can even embed the whole cannula underneath the choroidal tissues. We believe that it would lead to iatrogenic damage if the infusion were started in that case. The detached choroidal tissues can partially cover the inner opening of the cannulas, and the repeated in-and-out intravitreous maneuvers can push or drag the pars plana epithelium or vitreous base, causing iatrogenic ora serrata dialysis and/or retinal breaks. However, the potential of MIV systems to help solve these problems cannot be ignored if there is a well-designed surgical approach.
The advantages of MIV systems are well documented, covering a wide range of vitreoretinal diseases under complex conditions [20-23]. The trocar cannula system provides a reliable tool for easy and safe three-port sclerotomies. Nonetheless, the presence of the problems mentioned above sometimes limits the full usability of this tool. The most frequently recommended solutions are introducing a longer trocar $(6 \mathrm{~mm})$ and clearing the infusion tip with a MIV blade from another sclerotomy $[9-11,13]$. However, a longer trocar is not always available in some areas of the world (such as the authors' institution and other ophthalmic institutions in China because of local laws and regulations). Clearing the infusion tip with a MIV blade can cause sharp trauma to the lens and choroidal tissues, which increases surgical difficulty and worsens patient prognosis.

The essence of the surgical approach is maintaining IOP to prevent the collapse of the eye wall, creating and broadening the inner opening for the infusion cannula, and indenting the infusion trocar to allow for maximum visual exposure of the inner segment of the infusion trocar and its complete liberation in the vitreous cavity. The results suggest that the current approach enabled us to solve these challenging conditions with a step-by-step approach, without the need for sclerotomy or any other maneuvers related to the anterior chamber. Finally, the current approach strictly follows the rules of microincision and ensures the full usability of the 25-gauge vitrectomy pack as conversion to 20-gauge infusion or enlarged sclerotomy to allow the release of the SCF was not needed throughout the entire procedure.

There were several limitations that should be acknowledged. This is a consecutive case series study without a control group, and no other surgical techniques, such as a longer trocar or a MIV blade, were used to draw the conclusions.

\section{CONCLUSIONS}

The current approach is a feasible option to prevent inadvertent fluid/air perfusion during 
PPV in RRD eyes with extensive CD, SCF, and hypotony. Continuous refinement of the current method is expected in the future.

\section{ACKNOWLEDGEMENTS}

We would like to express our gratitude to all the participants, especially Prof. Wenjun Guo and Ms. Yu Yang, in the study for their kind assistance and cooperation.

Funding. This study, article processing charges and the open access fee for this study were funded by the grant from the Science and Technology Program of Guangzhou (201803010022).

Editorial Assistance. Writing support was provided by Peter Fan.

Authorship. All named authors meet the International Committee of Medical Journal Editors (ICMJE) criteria for authorship for this article, they take responsibility for the integrity of the work as a whole, and they have given their approval for this version to be published.

Disclosures. Zhaotian Zhang, Dong Fang, Manjuan Peng, Yantao Wei, Li Wang, Shuxin Fan and Shaochong Zhang have nothing to disclose.

Compliance with Ethics Guidelines. The study was approved by the institutional research committee of the Zhongshan Ophthalmic Center of Sun Yat-sen University (Guangzhou, China). All procedures performed on human participants were in accordance with the ethical standards of the institutional and/or national research committee and with the 1964 Helsinki Declaration and its later amendments or other comparable ethical standards. Informed consent was obtained from all participants included in the study.

Data Availability. The datasets generated and/or analyzed during the current study are available from the corresponding author upon reasonable request.
Open Access. This article is distributed under the terms of the Creative Commons Attribution-NonCommercial 4.0 International License (http://creativecommons.org/licenses/ by-nc/4.0/), which permits any noncommercial use, distribution, and reproduction in any medium, provided you give appropriate credit to the original author(s) and the source, provide a link to the Creative Commons license, and indicate if changes were made.

\section{REFERENCES}

1. Seelenfreund MH, Kraushar MF, Schepens CL, Freilich DB. Choroidal detachment associated with primary retinal detachment. Arch Ophthalmol. 1974;91:254-8.

2. Gottlieb F. Combined choroidal and retinal detachment. Arch Ophthalmol. 1972;88:481-6.

3. Sharma T, Challa JK, Ravishankar KV, Murugesan R. Scleral buckling for retinal detachment. Predictors for anatomic failure. Retina. 1994;14:338-43.

4. Li Z, Li Y, Huang X, et al. Quantitative analysis of rhegmatogenous retinal detachment associated with choroidal detachment in Chinese using UBM. Retina. 2012;32:2020-5.

5. Hawkins WR, Schepens CL. Choroidal detachment and retinal surgery. Am J Ophthalmol. 1966;62:813-9.

6. Brav SS. Serous choroidal detachment. Surv Ophthalmol. 1961;6:395-415.

7. Denwattana A, Prakhunhungsit S, Thoongsuwan S, Rodanant N, Phasukkijwatana N. Surgical outcomes of preoperative steroid for rhegmatogenous retinal detachment with associated choroidal detachment. Eye (Lond). 2018;32:602-7.

8. Wei Y, Wang N, Chen F, et al. Vitrectomy combined with periocular/intravitreal injection of steroids for rhegmatogenous retinal detachment associated with choroidal detachment. Retina. 2014;34:136-41.

9. Manayath GJ, Arora S, Kothari AR. 20-Gauge vitrectomy. In: Narendran V, Kothari AR, editors. Principles and practice of vitreoretinal surgery. 1st ed. New Delhi: Jaypee; 2014. p. 90-8.

10. Williamson TH. Introduction to vitreoretinal surgery. In: Williamson TH, editor. Vitreoretinal surgery. 2nd ed. Berlin: Springer; 2008. p. 21-59. 
11. Yang CM. Pars plana vitrectomy in the treatment of combined rhegmatogenous retinal detachment and choroidal detachment in aphakic or pseudophakic patients. Ophthalmic Surg Lasers. 1997;28:288-93.

12. Lakhanpal V, Schocket SS, Elman MJ, Nirankari VS. A new modified vitreoretinal surgical approach in the management of massive suprachoroidal hemorrhage. Ophthalmology. 1989;96:793-800.

13. Yoon YH, Sheu SJ, Terasaki H. Primary vitrectomy in rhegmatogenous retinal detachment. In: Schachat AP, editor. Ryan's retina. 6th ed. Amsterdam: Elsevier; 2018. p. 1933-40.

14. Oshima Y, Wakabayashi T, Sato T, Ohji M, Tano Y. A 27-gauge instrument system for transconjunctival sutureless microincision vitrectomy surgery. Ophthalmology. 2010;117:93-102.

15. Eckardt C. Transconjunctival sutureless 23-gauge vitrectomy. Retina. 2005;25:208-11.

16. Fujii GY, De Juan EJ, Humayun MS, et al. A new 25-gauge instrument system for transconjunctival sutureless vitrectomy surgery. Ophthalmology. 2002;109:1807-12, 13.

17. Wu Z, Ding N, Yu M, et al. Identification of potential biomarkers for rhegmatogenous retinal detachment associated with choroidal detachment by vitreous iTRAQ-based proteomic profiling. Int J Mol Sci. 2016;17:2052.

18. Dai Y, Wu Z, Sheng H, Zhang Z, Yu M, Zhang Q. Identification of inflammatory mediators in patients with rhegmatogenous retinal detachment associated with choroidal detachment. Mol Vis. 2015;21:417-27.

19. Yu Y, An M, Mo B, Yang Z, Liu W. Risk factors for choroidal detachment following rhegmatogenous retinal detachment in a Chinese population. BMC Ophthalmol. 2016;16:140.

20. Romano MR, Cennamo G, Ferrara M, Cennamo M, Cennamo G. Twenty-seven-gauge versus 25-gauge vitrectomy for primary rhegmatogenous retinal detachment. Retina. 2017;37:637-42.

21. Rizzo S, Barca F, Caporossi T, Mariotti C. Twentyseven-gauge vitrectomy for various vitreoretinal diseases. Retina. 2015;35:1273-8.

22. Sandali O, El SM, Lecuen N, et al. 25-, 23-, and 20-gauge vitrectomy in epiretinal membrane surgery: a comparative study of 553 cases. Graefes Arch Clin Exp Ophthalmol. 2011;249:1811-9.

23. Shah CP, Ho AC, Regillo CD, Fineman MS, Vander JF, Brown GC. Short-term outcomes of 25-gauge vitrectomy with silicone oil for repair of complicated retinal detachment. Retina. 2008;28:723-8. 